\title{
La démocratie participative et les devoirs du citoyen
}

\author{
Michel Forsé et Caroline Guibet Lafaye
}

Les études portant sur la citoyenneté privilégient souvent la question des droits des citoyens, qu'il s'agisse des droits-libertés (comme la liberté de conscience), des droitscréances (comme les droits sociaux relatifs à la santé ou à l'éducation) ou encore des droits objectifs face aux droits subjectifs (Schnapper, 2000). Peu d'entre elles s'interrogent sur le pendant de ces droits, c'est-à-dire sur les devoirs du citoyen. La question n'est certes pas nouvelle puisque des auteurs classiques comme Pufendorf (1673) s'y sont attelés depuis bien longtemps et le «devoir de civilité » qu'évoque Rawls de manière plus contemporaine s'inscrit dans cet héritage. Or un tel devoir est davantage moral que légal puisqu'il consiste à «expliquer aux autres comment, sur ces questions fondamentales, les principes et les programmes qu'ils défendent et pour lesquels ils votent peuvent être fondés sur les valeurs politiques de la raison publique » (Rawls, 1993, p. 264), c'est-à-dire sur des arguments qui constituent des raisons publiques justifiant les manières d'agir au sein de la sphère politique. Ce devoir s'impose aux législateurs comme à tout citoyen participant au forum public et prenant part aux élections. La citoyenneté doit donc aussi se comprendre comme une participation politique se traduisant, notamment, par le fait de s'impliquer dans les processus de décision politique et les possibilités d'influencer les choix collectifs. Pour le dire d'une autre manière, être citoyen à part entière ne correspond pas seulement à un état, à une relation d'appartenance fonctionnellement spécifiée et en quelque sorte passive entre l'individu et l'Etat ou la nation. C'est également un engagement en vertu duquel chacun est activement citoyen.

Cette citoyenneté «active», sur laquelle les recherches mettent aujourd'hui beaucoup plus l'accent (Gutmann et Thompson, 1997 ; Habermas, 1997 ; Blondiaux et Sintomer, 2002 ; Bévort, 2002), désigne une participation à la gouvernance et implique que les citoyens acceptent ou rejettent les raisons qu'eux-mêmes et leurs représentants offrent à l'appui des lois et des politiques qui les engagent mutuellement. Elle s'actualise factuellement dans nombre de processus et de procédés participatifs, tels que les conférences de citoyens, les conférences de consensus, les auditions publiques, etc. Autant d'instances où s'opère une « codétermination de politiques publiques » (Joss, 1999, p. 291) permettant aux citoyens de réguler de manière libérale leurs désaccords. C'est au total l'ensemble de ces implications qui donne son contenu à l'idée de « démocratie participative », que nous retiendrons dans cette étude, en son acception large de participation à la vie politique publique.

En regard du devoir de civilité qu'évoque Rawls, la question se pose alors de savoir quel lien existe entre la nature des jugements moraux convoqués au titre de justification de normes collectives (de normes de justice, par exemple) et les attitudes des individus-citoyens à l'égard de la démocratie participative. Cette question n'est cependant pas seulement théorique, elle est aussi empirique. Et c'est d'ailleurs uniquement sous cet angle que nous l'aborderons ici. En d'autres termes, dans les sociétés contemporaines qui sont les nôtres, existe-t-il un lien entre les devoirs que l'on assigne moralement au citoyen et les opinions sur la démocratie participative d'une part, tout comme, d'autre part, les formes de participation active (à la vie civique, politique ou aux prises de décision) qui sont pratiquées ou valorisées ? Pour apporter des éléments de réponse, nous aurons recours à l'enquête de l'International Social Survey Programme (ISSP) puisqu'il se trouve que, pour la première fois en 2004, elle 
était centrée sur le thème de la citoyenneté et que certaines des questions qu'elle comporte permettent de tester l'hypothèse d'un tel lien.

\section{L'instauration de la raison publique}

Avant que d'en venir à l'examen de ces données, le contexte et les enjeux théoriques de cette hypothèse doivent cependant être davantage situés et spécifiés. Le point de départ est à la fois simple et bien connu. Les sociétés modernes dont nous parlons se caractérisent par un pluralisme radical des conceptions du bien, des visions du monde et des valeurs qui y prévalent. Elles ne peuvent plus espérer fonder leur cohésion sur une de ces conceptions du bien qui se devrait d'y être partagée par tous ou presque. La solution perfectionniste des Anciens n'étant plus praticable, où se trouve le moteur de cette cohésion ? Peut-on espérer recourir à une forme ou une autre d'utilitarisme au titre de fondement ?

Ce serait oublier que la raison démocratique, qui fait le cœur de la vie politique, est irréductible au seul calcul d'intérêts. Les décisions prises dans la sphère politique publique le sont en référence à des principes de justice reconnus par tous. Pour reprendre la présentation qu'en donne Rawls, ces principes sont ceux que chacun accepterait s'il était placé dans les conditions du voile d'ignorance, c'est-à-dire dans l'ignorance de sa situation personnelle et sociale comme des caractéristiques et du contexte de sa société (Rawls, 1971, p. 168-169). Mais au-delà de cette situation abstraite et idéale (quoique régulatrice), lorsque dans les faits chacun est conscient de sa conception du bien, de ses talents, de ses intérêts et de sa propre situation sociale, comment l'accord qui émergeait derrière le voile d'ignorance autour de principes de justice relatifs à la structure de base de la société, peut-il persister ? Selon Rawls, il suffit pour cela que chacun reconnaisse et accepte les principes de la raison publique. Comme il l'écrit: "pour comprendre comment se conduire comme un citoyen d'une

démocratie, il faut comprendre l'idéal de la raison publique » (Rawls, 1993, p. 264). Etant donné le rôle fondamental que joue ce concept, arrêtons-nous un instant sur son contenu.

La raison publique consiste en une conception politique de la justice qui, dans nos démocraties constitutionnelles, assigne une priorité à des droits, à des libertés ainsi qu'à des possibilités de base. Elle garantit à tous les citoyens les moyens qui leur permettent d'avoir un usage effectif de leurs libertés et de leurs possibilités de base. La raison publique impose que les questions politiques fondamentales soient réglées en faisant appel aux valeurs politiques exprimées par cette conception de la justice, ce qui signifie que ce sont les valeurs politiques acceptées par la société politique qui règleront les questions relatives, par exemple, au droit de vote et à la religion, à l'égalité ou à la propriété. On attend, dans le règlement de ces questions, que les individus renoncent à imposer leurs valeurs personnelles et acceptent la prévalence des valeurs politiques sur celles-ci. Ceci étant, l'idéal de la raison publique commande seulement que les citoyens conduisent «leurs discussions fondamentales à l'intérieur du cadre constitué par ce que chacun considère comme une conception politique de la justice qui est fondée sur des valeurs dont on peut raisonnablement espérer que les autres y souscriront, chacun étant, en toute bonne foi, prêt à défendre cette conception ainsi comprise » (Rawls, 1993, p. 275). La raison publique signifie donc que, sur le forum public et dans les débats politiques, les valeurs politiques qu'elle incarne prévaudront sur toute doctrine compréhensive mais elle ne concerne pas nos délibérations et nos réflexions personnelles dans la sphère privée sur les questions politiques ni le raisonnement que tiennent, à leur sujet, les membres de diverses associations comme par exemple les Eglises ou les syndicats.

Dans ces conditions, Rawls considère que cet idéal de la raison publique est en mesure de susciter une adhésion sans exiger, de la part des personnes concernées, de souscrire intégralement à une conception libérale de la justice. Les principes qu'elle exprime font tout simplement l'objet d'un «consensus par recoupement»(Rawls, 1993, p. 211). Un tel consensus suppose que les individus trouvent, parmi leur conception du bien, des raisons 
d'adhérer à cet idéal sans que celui-ci ne soit, par exemple, inculqué aux individus par l'intermédiaire de l'école républicaine, promotrice des valeurs affirmées et défendues par la République.

Des auteurs comme J. Habermas soulignent cependant que Rawls présuppose l'accord autour de ces principes plus qu'il n'identifie les médiations par lesquelles y parvenir (Habermas \& Rawls, 1997, p. 158). Le consensus par recoupement se constate simplement comme un fait de la raison (publique). Or Habermas montre que cela est loin d'être suffisant. Selon lui, il est fondamental de déterminer comment ce consensus se construit et comment, une fois obtenu, il se perpétue. Comme cette élaboration ne saurait se passer des procédures de discussion qu'illustrent les débats publics institutionnalisés, le dialogue et les institutions qui promeuvent le débat jouent un rôle central dans la constitution du consensus. La démocratie délibérative ou participative est en somme l'instrument même de l'instauration de la raison publique.

D'où cette citoyenneté active dont nous parlions plus haut et qui se manifeste dans des comportements liés au processus électoral (inscription sur les listes électorales, participation à une campagne), mais aussi au fait de se tenir informé de la vie politique, de prendre part à des discussions politiques, d'assister à des réunions politiques, de contacter un élu, d'adhérer à un parti, voire plus largement de participer à des actions qualifiées de protestataires (signer une pétition, prendre part à une manifestation, occuper un bâtiment public, etc.). Dans tous ces cas, les citoyens adoptent à l'égard du «monde social» et de ses normes, une posture réflexive et par conséquent critique. Cette dernière, en coïncidant avec le point de vue «prééminent-à-la-société » (Kohlberg, 1981), incarne l'attitude d'un participant à la discussion. Le dialogue interroge et thématise, puis légitime ou invalide les exigences de validité attachées aux normes sociales.

Selon Habermas, cette discussion, tout comme la participation politique, se comprend, au plan théorique et normatif, dans le sens d'une formation démocratique de la volonté, i.e. d'une formation de l'opinion et de la volonté par le dialogue et l'argumentation. Cette formation de la volonté, constitutive du processus démocratique, est conditionnée par la reconnaissance de droits politiques fondamentaux aux citoyens. Elle dresse, corrélativement, le cadre au sein duquel l'autonomie politique de ces derniers s'exerce et à travers lequel ils instaurent un droit légitime (Habermas, 1997, p. 140). Quoiqu'on puisse parfois l'oublier la délibération publique est une dimension essentielle de la démocratie, plus importante encore que son aspect participatif incarné notamment par le vote (Sen, 2006, p. 16-17). Elle n'est d'ailleurs pas une « invention de l'Occident» mais plutôt la meilleure façon de respecter les convictions de chacun s'exprimant sur la scène publique. Cette citoyenneté «participante », dont les fondements normatifs sont donnés par l'usage public de la raison et par la délibération publique, est indissociable d'une «éthique publique démocratique », en ce sens que, pour ces agents autonomes que sont les citoyens, la discussion est partie prenante de la légitimité politique (cf. Audard, 2007, p. 76). En d'autres termes, la participation des citoyens aux débats conduit à la légitimation de ce qui est, politiquement, en débat.

Les individus impliqués dans ces processus participatifs - et parfois délibératifs mettent en effet en œuvre leur capacité normative, en formulant des jugements normatifs sur les questions évoquées ou les normes publiques (éthiques et/ou politiques) en jeu. Ce faisant s'élaborent, dans leur conscience morale, des raisons d'adhérer ou de rejeter les normes collectives. On a souvent constaté que les personnes engagées dans et par ces décisions peuvent être conduites à accepter, dans le cadre de procédures participatives, des résolutions qui ne sont pas en parfaite congruence avec leur point de vue. A tout le moins, elles ne s'y opposent plus de manière rédhibitoire (Urfalino, 2007). Ainsi l'extension des modèles procéduraux aux querelles morales contribue à étendre la légitimité des décisions aux yeux des personnes concernées et de celles qui ne le sont pas directement, dans la mesure où 
l'impartialité y joue un rôle décisif et où ces processus d'intercompréhension reposent sur des considérations éthiques relatives à la justice des procédures comme au traitement des acteurs.

$\mathrm{Au}$ sein de cette «querelle de famille », que nous venons de résumer, entre tenants de la position de Rawls et ceux suivant plutôt Habermas, il semble qu'il soit en fait possible d'identifier un terrain de convergence. Certes, d'un côté, on ne peut se contenter de constater le consensus ou de présupposer l'accord. Il doit se construire et il n'est d'ailleurs jamais définitivement acquis. Mais, de l'autre, peut-on penser que la simple participation à la discussion suffit à ce que le sujet fasse siennes les décisions qui auront éventuellement été prises ? Ne faut-il pas avec Rawls insister aussi sur la nécessaire adhésion de ce sujet à l'idéal de la raison publique ? Comme personne ne nie que cette adhésion passe, dans une large mesure, par une participation aux processus politiques, il est en tous cas clair pour chacun des protagonistes de ce débat que l'idéal de la raison publique engage un idéal de participation démocratique. Or, c'est seulement ce lien, dont nous avons fait l'hypothèse plus haut, que nous souhaitons ici examiner dans sa dimension empirique. Cet examen est rendu possible par le fait que l'idéal de la raison publique, que Rawls relie aux devoirs de civilité et qui fait l'objet de jugements normatifs de la part des membres de la communauté politique, peut être cerné empiriquement par la manière dont ces derniers conçoivent l'idéal du citoyen.

Pour procéder à cet examen, nous commencerons par identifier, au travers de tout un ensemble de questions posées dans l'enquête de l'ISSP, les devoirs moraux du «citoyen idéal » en nous demandant si une typologie stable se dégage des réponses. Nous envisagerons ensuite la façon dont les enquêtés comprennent aujourd'hui la « démocratie participative » et comment, selon leurs manières d'envisager les devoirs du citoyen, le sens conféré et les obligations corrélées à cette forme de démocratie varient. Nous exploiterons enfin les questions relatives aux formes de participation à la vie civique ou politique (manifestations, pétitions, etc.) ou encore à la prise de décision, en nous demandant à nouveau dans quelle mesure les réponses varient selon les types de citoyen idéal auxquels les personnes sondées adhèrent ${ }^{1}$.

\section{Les devoirs du citoyen selon les pays et les catégories sociales}

La comparaison internationale qu'autorise l'enquête de l'ISSP est de ce point de vue un atout et même un impératif si l'on veut pouvoir discerner le général de la singularité nationale. Pour cette étude, parmi tous les pays participant à ce programme, nous en avons sélectionné sept en Europe. Ils représentent ses grandes aires géographiques: au nord, la Suède (taille d'échantillon $n=1295)$, au sud l'Espagne $(n=2481)$, entre les deux la GrandeBretagne $(n=853)$, la France $(n=1419)$, l'Allemagne $(n=1332)$ et la Suisse $(n=1078)$, et à l'est la Pologne $(n=1277)$. Pour faire la part de ce qui pourrait s'avérer être trop lié au contexte européen, nous avons également retenu les États-Unis $(n=1472)$ et le Japon $(n=$ 1343). Dans chaque cas, il s'agit d'un sondage aléatoire effectué auprès de personnes âgées de 18 ans ou plus. Au total, 12550 individus ont été interrogés ${ }^{2}$.

\footnotetext{
${ }^{1}$ Les thèmes abordés dans le questionnaire sont assez nombreux mais ne couvrent évidemment pas l'ensemble des questions que l'on peut se poser à propos de la citoyenneté. En particulier, les problèmes liés à l'identité nationale, à la place des étrangers et des diverses communautés ethniques avaient fait l'objet de l'enquête de 2003 et ne sont donc pas repris en 2004. Les opinions recueillies à cette dernière date portent notamment sur le citoyen idéal, les libertés publiques (civiles ou politiques), la justice sociale, la démocratie participative, différentes formes d'actions collectives, la confiance dans les autres ou dans les institutions, le fonctionnement de la démocratie et des institutions publiques. Certaines pratiques sont également abordées, par exemple dans les domaines de la protestation, de la sociabilité ou de la participation sociale.

${ }^{2}$ Chaque pays a fourni une variable de redressement que nous avons utilisée (après quelques corrections mineures). Mais nous avons également calculé une variable de pondération tenant compte de la taille effective de la population âgée de 18 ans ou plus dans chaque cas (ce qui laisse toutefois inchangé le nombre total d'individus enquêtés).
} 
L'enquête commence par une série de dix questions ayant trait à ce que devrait faire un «bon citoyen » aux yeux de l'interviewé. Pour chacune de ces questions, il lui était demandé de situer sa réponse sur une échelle allant de 1 , pour « pas important du tout », à 7 pour «très important ». C'est d'ailleurs le cas pour beaucoup des questions de ce questionnaire qui, de ce fait, n'appellent pas à des jugements trop tranchés et permettent au contraire à l'enquêté de nuancer ses réponses. Ces dix questions sont les suivantes. "Selon vous, un bon citoyen devrait ?»: 1 - toujours voter aux élections, 2 - ne jamais chercher à frauder le fisc, 3 toujours obéir aux lois et règlements, 4 - garder un œil sur ce que fait le gouvernement, 5 être actif dans des associations sociales ou politiques, 6 - essayer de comprendre les raisons de ceux qui m'ont pas les mêmes opinions, 7 - choisir d'acheter des produits pour des raisons éthiques, politiques ou environnementales, même s'il coûtent un peu plus chers, 8 - être prêt à aider les personnes démunies dans son pays, 9 - être prêt à aider les personnes démunies dans le monde, 10 - être prêt à servir dans l'armée en cas de besoin.

Posées de cette façon, ces questions réfèrent à la sphère publique et non privée. Il ne s'agit pas de savoir comment se comporter dans ses relations personnelles, mais de juger de ce que doit faire un citoyen. Elles permettent donc d'élucider le type d' « éthique publique démocratique » que les enquêtés associent à la «citoyenneté idéale ». Et puisqu'il s'agit d'idéal, il est légitime de considérer que leurs réponses manifestent une attitude réflexive sur le «monde social » (c'est-à-dire le point de vue «prééminent-à-la-société ») qui peut être rapportée à l'attitude hypothétique d'un participant à une argumentation, thématisant les exigences de validité normatives correspondantes.

Tableau 1 - Ce que devrait faire un bon citoyen selon les pays

\begin{tabular}{|c|c|c|c|c|c|c|c|c|c|c|c|}
\hline & $\mathrm{DE}$ & GB & US & $\mathrm{SE}$ & PL & $\mathrm{JP}$ & ES & FR & $\mathrm{CH}$ & Moy. $^{\text {a }}$ & Moy. $^{b}$ \\
\hline $\begin{array}{l}\text { Toujours voter aux } \\
\text { élections }\end{array}$ & 5,17 & 5,27 & 6,20 & 6,27 & 5,56 & 5,79 & 5,81 & 6,19 & 5,42 & 5,78 & 5,85 \\
\hline $\begin{array}{l}\text { Ne jamais frauder } \\
\text { le fisc }\end{array}$ & 5,82 & 6,30 & 6,39 & 5,71 & 6,02 & 6,44 & 6,09 & 5,86 & 5,72 & 6,04 & 6,22 \\
\hline $\begin{array}{l}\text { Toujours obéir aux } \\
\text { lois }\end{array}$ & 5,98 & 6,46 & 6,47 & 5,96 & 6,45 & 6,28 & 6,17 & 5,85 & 5,50 & 6,13 & 6,29 \\
\hline $\begin{array}{l}\text { Surveiller l'action } \\
\text { gouvernementale }\end{array}$ & 5,29 & 5,18 & 6,13 & 5,94 & 5,60 & 5,66 & 5,23 & 5,18 & 5,01 & 5,47 & 5,69 \\
\hline $\begin{array}{l}\text { Participer aux } \\
\text { associations }\end{array}$ & 3,74 & 3,38 & 4,63 & 3,43 & 4,23 & 3,85 & 4,46 & 4,30 & 4,19 & 4,10 & 4,20 \\
\hline $\begin{array}{l}\text { Comprendre les } \\
\text { raisons des autres }\end{array}$ & 5,56 & 5,63 & 5,83 & 5,73 & 5,58 & 5,30 & 5,80 & 5,65 & 5,80 & 5,67 & 5,66 \\
\hline $\begin{array}{l}\text { Choisir des produits } \\
\text { éthiques }\end{array}$ & 4,38 & 4,29 & 4,83 & 4,55 & 3,85 & 4,33 & 5,36 & 4,39 & 4,91 & 4,63 & 4,58 \\
\hline $\begin{array}{l}\text { Aider les démunis } \\
\text { dans le pays }\end{array}$ & 5,55 & 5,23 & 6,04 & 5,13 & 5,88 & 5,11 & 6,05 & 5,36 & 5,59 & 5,61 & 5,67 \\
\hline $\begin{array}{l}\text { Aider les démunis } \\
\text { dans le monde }\end{array}$ & 4,80 & 4,64 & 4,88 & 4,78 & 5,44 & 4,59 & 5,82 & 4,64 & 5,18 & 5,06 & 4,87 \\
\hline $\begin{array}{l}\text { Servir dans l'armée } \\
\text { si besoin }\end{array}$ & 3,95 & 4,50 & 5,40 & 4,27 & 5,50 & 3,08 & 3,70 & 4,14 & 4,05 & 4,26 & 4,55 \\
\hline
\end{tabular}

a : Moyenne sans pondération démographique.

$\mathrm{b}$ : Moyenne avec prise en compte du poids démographique de chaque pays.

NB : Dans chaque pays les réponses se font sur une échelle allant de 1 pour «pas important du tout » à 7 pour «très important ».

Source : ISSP 2004. 
Comme le montre le tableau 1, les personnes interrogées ont en moyenne jugé que chacun de ces devoirs était important (les moyennes sont toujours supérieures à 4). Obéir aux lois et ne pas frauder le fisc apparaissent cependant prioritaires (les scores moyens sont supérieurs à 6). Toujours voter aux élections, comprendre les raisons des autres, garder un œil sur ce que fait le gouvernement et aider les plus démunis dans son pays suivent de près ces deux premières modalités. Aider les moins privilégiés dans le monde suscite un peu moins d'engouement mais ce sont surtout les items « choisir d'acheter des produits pour des raisons éthiques, politiques ou environnementales, même s'ils coûtent un peu plus chers », "être prêt à servir dans l'armée en cas de besoin » et «être actif dans les associations sociales ou politiques » qui apparaissent (relativement) moins populaires.

D'un pays à l'autre, les différences sont, au terme d'une analyse de variance, significatives. Toutefois, dans chaque pays, la hiérarchie de ces différents devoirs reste proche de celle qui vient d'être constatée pour l'ensemble. Les écarts les plus notables concernent le fait de toujours voter aux élections qui est moins important pour les Allemands et les Suisses. L'obéissance aux lois l'est également moins pour ces derniers tout comme, dans une moindre mesure, la surveillance de l'action du gouvernement. L'achat de produits plus chers pour des raisons éthiques séduit peu les Polonais mais, à l'instar des Américains, ils se disent davantage prêts à servir dans l'armée si besoin. Les Espagnols se signalent quant à eux par une plus forte propension à penser qu'un bon citoyen devrait aider les personnes en difficulté dans son pays.

Comme nous l'avons rappelé plus haut en note, le questionnaire s'étend parfois à des pratiques (déclarées mais non observées). Certaines peuvent alors être mises en regard des opinions qui leur correspondent afin de déterminer si elles sont suivies d'effets. Or, de ce point de vue, il s'avère qu'il y a le plus souvent une forte corrélation entre les deux. Prenonsen quelques exemples en nous limitant aux manières de dessiner les contours du citoyen idéal. Ceux qui estiment qu'il devrait participer à des associations se déclarent eux-mêmes actifs dans des associations. Ceux qui jugent qu'il devrait toujours voter ne sont pas abstentionnistes. Ceux qui pensent qu'il devrait toujours obéir aux lois ne se joignent guère aux actions de boycott de certains produits (la corrélation reste négative mais non significative au Japon et en Pologne) et, dans les pays où le lien est significatif, ils ne tentent pas d'agir contre une loi injuste. Ceux qui souhaitent que le bon citoyen aide les moins privilégiés, que ce soit dans son pays ou dans le monde, ont davantage tendance à donner de l'argent ou à en collecter, même si le questionnaire n'évoque explicitement à ce sujet qu'un motif « social ou politique » et non par exemple humanitaire (lorsqu'il s'agit d'une aide limitée au pays, la corrélation n'est pas significative au Japon et en Pologne). Ceux qui pensent que le citoyen idéal devrait chercher à comprendre les raisons des autres participent volontiers à des forums de discussion sur l'Internet ou auraient pu le faire - probablement, mais on ne peut le vérifier ici, s'ils étaient équipés. En France et en Espagne, seuls pays de notre panel où ces questions optionnelles ont été posées, ils trouvent également très important (7 sur une échelle de 1 à 7) lorsqu'ils rencontrent des gens pour la première fois, « de faire ou de dire quelque chose pour montrer qu'ils les respectent » ou lorsqu'il s'agit de personnes avec lesquelles ils sont en profond désaccord, « de faire ou de dire quelque chose pour leur manifester leur tolérance ». Ils participent souvent à des discussions politiques avec des proches et essayent de les persuader lorsqu'ils ont une conviction forte. Le plus souvent, ce comportement se retrouve d'ailleurs encore davantage parmi ceux qui estiment que le bon citoyen devrait garder un œil sur ce que fait le gouvernement.

Même s'il n'est pas possible ici de faire la part de ce qui relève d'une possible rationalisation a posteriori, il n'y a donc pas de décalages notables entre les opinions sur le citoyen idéal et les pratiques effectives correspondantes de ceux qui émettent ces opinions. 
Parmi les variables sociodémographiques, l'âge est celle qui a le plus systématiquement un effet sur les conceptions du citoyen idéal. Les plus âgés trouvent davantage que les plus jeunes qu'il faut se conformer aux lois ou aux normes (toujours voter), ne pas chercher à frauder le fisc, participer aux associations, aider les moins privilégiés dans le pays (alors que dans le monde, lorsque l'âge a un effet significatif, il s'agit plutôt des plus jeunes), être prêt à rejoindre l'armée ou garder un œil sur l'action du gouvernement. Quelques pays peuvent faire exception à ces corrélations selon les questions (par exemple l'âge joue peu s'agissant de choisir des produits pour des raisons éthiques aux Etats-Unis, en Suède, Pologne, Espagne et France), mais ces exceptions sont au total très peu nombreuses. En revanche, l'âge n'a pas d'effet significatif pour ce qui est de chercher à comprendre les raisons d'autrui.

Le sexe n'en a pas davantage (sauf en Suède et en Espagne où les femmes surclassent les hommes) mais c'est une situation beaucoup plus générale pour cette variable avec, là aussi, quelques exceptions. Les femmes trouvent, dans quasiment tous les pays, plus important que les hommes que le bon citoyen suive les lois, y compris en ce qui concerne le fisc, ou aide les plus démunis dans son pays ou dans le monde. Aux Etats-Unis, en Suède et en Espagne, elles affirment plus volontiers qu'il devrait choisir des produits éthiques, tandis qu'à l'inverse, dans chaque pays, les hommes pensent plutôt qu'il devrait être prêt à servir dans l'armée en cas de besoin. Pour le reste, le rôle du sexe n'apparaît pas significatif.

Il en va à peu près de même du positionnement politique. On peut tout au plus noter ici que s'agissant d'aider les plus démunis, lorsque le lien est significatif, il s'agit plutôt d'une opinion de gauche mais que «servir dans l'armée » est mieux corrélé avec une opinion de droite. Toujours voter aux élections n'est pas lié à une opinion partisane, et dans les trois pays où c'est le cas, cette opinion varie : elle se situe à gauche aux Etats-Unis, au centre en Suède et à droite en France.

Participer ou simplement adhérer à une association intervient davantage et de manière plus régulière sur les conceptions du bon citoyen. Cette pratique conduit à trouver plus important que la moyenne les items : toujours voter aux élections ou obéir aux lois, y compris en matière fiscale, surveiller l'action gouvernementale, comprendre les raisonnements des autres, choisir des produits pour des raisons éthiques et bien évidemment participer aux associations. En revanche, «aider les moins privilégiés » et «servir dans l'armée » ne sont pas liés, ou rarement, à la participation sociale effective.

L'auto-position dans la hiérarchie sociale ${ }^{3}$ et le niveau de diplôme ont des effets ou absences d'effets souvent congruents. Dans la quasi-totalité des pays, se situer à des niveaux élevés dans la hiérarchie sociale ou avoir un haut niveau de diplôme conduisent significativement à penser qu'il est important que le citoyen participe à des associations, vote aux élections, cherche à comprendre les raisonnements des autres ou opte pour des produits pour des raisons éthiques, politiques ou environnementales. Surveiller l'action du gouvernement suit la même logique, à ceci près que le diplôme a moins souvent un effet significatif. L'aide aux plus démunis dans le pays n'est en général pas sensible à l'une ou l'autre de ces deux variables. Lorsque c'est le cas (en Grande-Bretagne, Suède, Japon et Espagne), ce sont cette fois les moins diplômés ou ceux qui se positionnent eux-mêmes au bas

\footnotetext{
${ }^{3}$ Le code ISCO (International Standard Classification of Occupations) dont nous disposons dans cette enquête pour classer les professions n'a pas la même nature hiérarchique, au moins pour les salariés, que par exemple la PCS (Professions et Catégories Socioprofessionnelles) utilisée en France. Ce code classe en effet autant les professions que les secteurs d'activité. L'auto-positionnement sur une échelle socio-économique (allant de 1 à 10 dans le questionnaire) approxime cependant assez fidèlement la place dans la hiérarchie sociale effective. Cette auto-position est en particulier bien corrélée avec les niveaux de revenu et la place de la profession de l'interviewé dans une échelle de type PCS ou de prestige. Pour indiquer et résumer la position sociale de l'enquêté, nous utiliserons donc son auto-positionnement en regroupant toutefois les valeurs de l'échelle qui lui était proposée par deux pour ne plus conserver que cinq niveaux (de 1 pour le plus élevé à 5 pour le plus bas). Cette question n'est malheureusement pas posée en Grande-Bretagne.
} 
de la pyramide sociale (États-Unis) qui y adhèrent le plus. A l'inverse, le souhait d'étendre cette aide au monde entier touche d'abord les plus diplômés et, en France et en Suisse, les positions sociales élevées ou, en Pologne, moyennes (ailleurs l'effet de la position sociale n'est pas significatif). L'obéissance aux lois relève d'un modèle plus complexe. La place dans la hiérarchie sociale a le plus souvent un effet mais il varie selon les pays. Le niveau de diplôme n'a pas de conséquence significative aux États-Unis, en Pologne et au Japon, tandis qu'ailleurs cette obéissance recueille surtout les faveurs des moins diplômés. L'effet de la position sociale est plus rare en ce qui concerne l'opinion sur la fraude fiscale. Il ne s'observe de manière nette qu'en Suède où elle est surtout le fait des positions les moins élevées. Le diplôme intervient moins souvent que lorsqu'il s'agit de l'obéissance plus générale aux lois, mais lorsque c'est le cas (Suède, France, Suisse), ce sont à nouveau les moins diplômés qui souhaitent que le bon citoyen respecte la loi en matière fiscale.

Malgré d'inévitables exceptions, finalement peu nombreuses, les diverses catégories sociodémographiques ont donc au total des effets qui vont plutôt dans le même sens d'un pays à l'autre. Une telle régularité incite à se demander si une typologie du citoyen idéal, valant pour l'ensemble des pays, ne pourrait pas se dégager de ces données. Pour rechercher si cette typologie est possible, nous aurons recours à des analyses en composantes principales $\left(\mathrm{ACP}^{4}\right)$.

\section{Quatre types de citoyen idéal}

Le premier axe de l'analyse en composantes principales, effectuée sur les dix variables d'opinion sur le citoyen idéal, correspond à un classique «effet taille». Toutes les fortes valeurs de ces variables (donc pour les modalités «important» ou «très important») se retrouvent du même côté de ce premier axe principal. Autrement dit, les fortes valeurs vont, sans exception, avec les fortes valeurs, et les faibles, avec les faibles. Les dix vecteurs représentant chacun une question ont la même orientation. C'est là un premier résultat massif et qui doit être souligné : les réponses à propos du bon citoyen sont positivement corrélées entre elles. Si l'on préfère, en moyenne, les enquêtés n'ont pas eu tendance à répondre « important » à une question et «pas important» à une autre. Mais, pour massif que soit ce premier résultat, il ne nous aide guère à construire une typologie puisque cela implique au contraire de pouvoir distinguer des modèles de réponses les uns des autres.

L'examen des axes factoriels suivants, en l'occurrence des axes 2 et 3, va cependant permettre d'y parvenir ${ }^{5}$. Sur le plan factoriel croisant ces axes, on voit cette fois clairement se dessiner des regroupements et des oppositions entre variables. Néanmoins, ces corrélations positives ou négatives ne sont pas toujours les mêmes d'un pays à l'autre et cela est dû en particulier à ce que la participation aux associations et le fait d'être prêt à servir dans l'armée ne sont pas toujours liés avec les mêmes autres variables. On aboutit au contraire à un résultat stable si l'on retire ces deux variables de l'analyse factorielle. C'est donc ce résultat que nous allons examiner en détail. Il est reproduit au graphique 1 pour l'ensemble des pays simultanément. On parvient en effet à un graphique factoriel quasiment identique dans chaque pays. Il n'y a à vrai dire qu'une seule exception pour une seule question au Japon : «toujours

\footnotetext{
${ }^{4}$ Rappelons que les enquêtés devaient pour chaque question sur le «bon citoyen » situer leur choix sur une échelle allant de 1 pour «pas important du tout » à 7 pour «très important». L'ACP tient ces réponses pour ordinales ou numériques. Dans le plan factoriel, chaque question est dès lors représentée par un vecteur dont l'orientation correspond aux plus fortes valeurs. Autrement dit, ici, le sens d'un vecteur pointe vers les réponses de type «très important»; les réponses de type «pas important du tout» se situant à l'opposé. Les axes factoriels sont rangés par ordre décroissant de variance totale du nuage de points qu'ils expliquent. Leur orientation est arbitraire.

${ }^{5} \mathrm{Au}$-delà du troisième axe, les autres composantes principales n'expliquent plus qu'une faible part de la variance totale et leur interprétation sociologique est plus délicate. Nous ne les examinerons donc pas ici. On peut par ailleurs rappeler que les axes 2 et 3 d'une ACP correspondent aux axes 1 et 2 d'une analyse de correspondances multiples - analyse que l'on applique lorsque les variables sont nominales.
} 
voter aux élections »y est proche de l'obéissance aux lois alors que, partout ailleurs, cette opinion se rapproche de "garder un œil sur l'action du gouvernement ». Puisqu'il n'y a que cette seule petite exception, on peut s'en tenir pour un commentaire valant quel que soit le pays à l'interprétation du graphique d'ensemble. Or, comme cela apparaît nettement sur ce graphique, l'opinion sur le citoyen idéal s'organise partout autour de quatre grands pôles.

1 - Au sud-est, l'aide au plus démunis dans le pays et dans le monde définit un type que l'on peut qualifier d'altruiste.

2 - Au sud-ouest, l'obéissance aux lois et ne pas frauder le fisc forme un type légaliste.

3 - Au nord-ouest, toujours voter aux élections et surveiller l'action gouvernementale se regroupent pour former un type que nous qualifierons, nous verrons pourquoi plus loin, d'individualiste indépendant.

4 - Au nord-est enfin, chercher à comprendre les raisons des autres et choisir un produit pour des raisons éthiques, permettent de parler d'un type pluraliste raisonnable ou libéral'.

\section{Graphique 1 - Analyse en composantes principales des opinions sur le citoyen idéal}

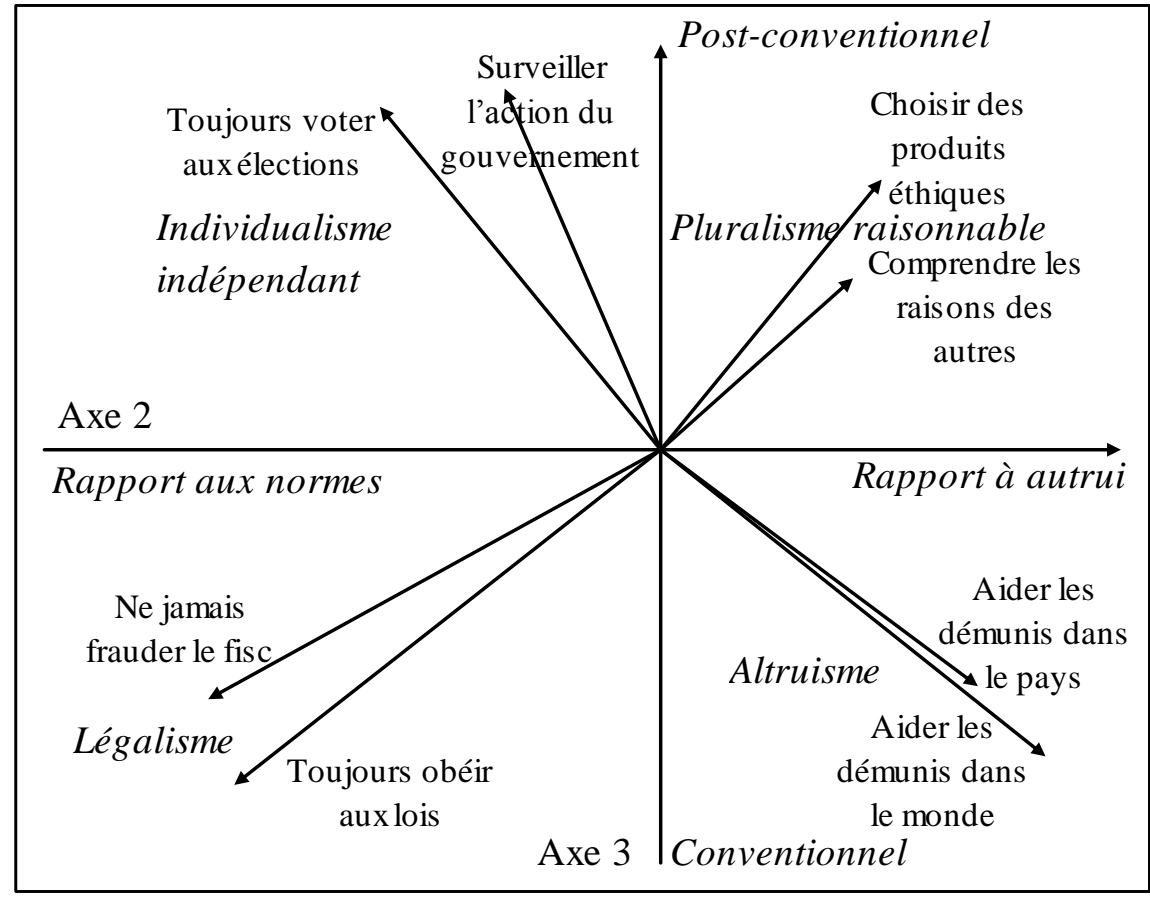

NB : les significations attribuées aux axes et aux quadrants sont en italique.

Avant d'examiner les raisons pour lesquelles on peut nommer ainsi chacun des types, précisons que, sous un angle méthodologique, il ne faut pas surinterpréter les rapprochements (angles aigus entre vecteurs dans le plan factoriel), les indépendances (angles droits) ou les oppositions (angles obtus) entre questions apparaissant au graphique 1. Le premier axe principal nous a montré que toutes les réponses à ces questions étaient positivement corrélées. En moyenne, les enquêtés ont jugé que tous ces items étaient importants pour définir un bon citoyen. Mais au-delà de ce résultat brut, ils les ont hiérarchisés différemment. C'est ce résultat relatif que met en évidence le graphique 1. Par exemple, les altruistes du type 1 ont accordé plus d'importance à l'aide aux plus démunis, un peu moins et à peu près autant aux

\footnotetext{
${ }^{6}$ Nous emploierons dans la suite ces deux termes indifféremment puisqu'ils reviennent au même. Précisons toutefois, s'il en était besoin, que nous faisons référence au «libéralisme », non pas en son sens français de libéralisme économique, mais au sens que ce terme a trouvé dans la philosophie morale et politique anglosaxonne à la suite de travaux trouvant leur origine au XVIIe siècle.
} 
questions relevant des types 2 et 4, et encore moins aux questions du type 3. Cela ne signifie pas qu'ils ont jugé de manière brute que les items des types 3, 2 ou 4 n'étaient pas importants. En revanche, ils les ont trouvés relativement moins importants que ceux du type 1 et selon l'ordre qui vient d'être donné. C'est seulement de ce profil relatif, où l'on met un peu plus l'accent sur l'importance de certains items et un peu moins sur d'autres, dont il est ici question. Au-delà du fait que tout le monde trouve que tout est important, ce sont bien ces profils relatifs qui nous intéressent et qui permettent de dégager la typologie qui vient d'être esquissée.

Parallèlement aux quatre types de citoyen idéal, les axes factoriels 2 et 3 peuvent eux aussi recevoir une interprétation. On sait que dans une analyse factorielle les axes n'ont pas d'autre signification a priori que statistique. Mais leurs relations avec les différents vecteurs représentés au graphique 1 (correspondant à chaque question) peuvent permettre de leur trouver un sens sur le fond. En fait, le repérage de la logique sous-jacente à chaque groupe va de pair avec le repérage de la signification des axes factoriels, en particulier ici où leur croisement est constitutif des quatre types qui se dégagent.

Le sens de l'axe horizontal 2 est assez peu problématique. Il oppose ceux qui, à droite sur le graphique 1, mettent l'accent sur le rapport du citoyen aux normes, lois ou institutions, qu'elles soient à respecter ou à questionner, à ceux qui, à gauche, mettent davantage l'accent sur le rapport à autrui (aider les autres en difficulté y compris par exemple par le moyen du commerce équitable, ou comprendre les autres). La signification de l'axe vertical 3 est en revanche beaucoup moins évidente.

Pour lui trouver un sens, il semble qu'une analogie partielle avec la théorie du développement moral de Kohlberg (1981) puisse être envisagée. D’après le résumé qui en est donné en annexe, il est bien certain que cette théorie ne peut être littéralement et totalement convoquée pour rendre raison ni pour comprendre la nature de la représentation du citoyen idéal à partir des questions étudiées ici. Il est toutefois possible de l'exploiter heuristiquement dans une perspective strictement analytique. Autrement dit, on peut en reprendre certains aspects tout en laissant ceux qui ne sauraient valoir dans notre contexte. En particulier, nous laisserons résolument de côté sa dimension naturaliste, tout comme la notion d'apprentissage constructif dont Piaget (1932) fait aussi usage, mais surtout nous ne retiendrons pas l'idée que nous avons affaire à des stades du développement moral s'articulant en une suite hiérarchisée et ordonnée, invariante et irréversible de structures discrètes. Sans entrer dans les longs débats que cette thèse a suscités, remarquons seulement que cette notion de «stades » suppose une évolution et une dynamique en deçà de laquelle demeure bien évidemment les données dont nous traitons. En somme, nous ne prétendons en aucune manière identifier ici des «stades » et ne conserverons des conclusions de Kohlberg que leur logique d'identification de types. Néanmoins, puisque c'est bien à la formulation de jugements moraux que sont appelés les enquêtés pour préciser la manière dont ils appréhendent le «bon» citoyen, il est légitime de se demander s'ils ne correspondent pas à certains des jugements que Kohlberg a repérés dans ses propres investigations empiriques sur la morale. Notre propos va alors essentiellement consister à montrer que l'axe 3 de notre ACP peut s'interpréter comme le passage de ce que Kohlberg nomme le «conventionnel» au «post-conventionnel»-interprétation qui bien évidemment se répercute sur celle de chacun des types.

On pourrait bien sûr utiliser d'autres termes, par exemple en distinguant sur cet axe entre loyauté (au sud) et légitimité (au nord). Les «altruistes» et les «conformistes» manifestent une loyauté à l'égard d'autrui ou à l'égard des normes, tandis que les «pluralistes libéraux » et les «individualistes indépendants » agissent pour leur part conformément à des principes au sujet desquels se pose, de façon problématique, la question de leur légitimité. Mais c'est au fond une autre façon de parler de la même chose et qui a le désavantage de masquer le rapport avec d'autres données sur les jugements moraux. 


\section{Du conventionnel au post-conventionnel}

En en restant donc au cadre conceptuel proposé par Kohlberg, remarquons tout d'abord que les stades pré-conventionnels 1 et 2 ne sont pas représentés ici pour la bonne et simple raison qu'aucune des questions posées ne leur correspond. Les auteurs du questionnaire n'ont évidemment pas envisagé de demander aux enquêtés d'identifier le bon citoyen à partir de sa peur de la punition ou de son égoïsme. La chose est bien normale. Par définition, puisqu'il s'agit de citoyenneté, c'est-à-dire au minimum du rapport à une communauté et à ses conventions, ce qui relève du pré-conventionnel ne peut apparaître.

En revanche, il semble bien que les «altruistes » qui mettent l'accent sur l'aide aux moins privilégiés dans leur pays, puis dans le monde, soutiennent un raisonnement qui au moins par certains aspects s'apparente au stade 3 de la conscience morale selon Kohlberg. Rappelons qu'il ne s'agit plus seulement à ce stade de respecter les règles mais de répondre aux attentes des proches ou des partenaires de l'interaction. L'attitude juste - i.e., dans notre perspective, celle qui fait que l'on est un bon citoyen - consiste en une interprétation des exigences de la justice à partir des attentes des autres. L'individu considère qu'il fait ce qui est juste, lorsqu'il vit conformément à ce que ses proches espèrent de lui ou lorsqu'il vit conformément à ce que les gens espèrent d'un fils, d'une sœur, d'un ami, etc. Il est vrai que cette dimension d'un autrui familier ou de proches n'intervient pas au travers des questions dont nous traitons. Néanmoins et si l'on admet simplement l'existence d'une analogie entre la figure de l'« altruiste » qui se dégage des réponses à ces questions et le troisième stade décrit par Kohlberg, cette référence demeure pertinente car ce qui prédomine ici c'est le fait « d'être bon ", c'est-à-dire d'avoir de bonnes intentions et de se sentir concerné par les autres, notamment s'ils sont démunis.

L'interprétation de l'attitude "légaliste » de notre deuxième type (toujours obéir aux lois, y compris en matière fiscale) à partir de la description kohlbergienne du stade 4 de la conscience morale apparaît sans doute plus pertinente encore. Kohlberg a montré qu'à ce stade la dimension du respect des lois et de l'obéissance aux normes était prédominante. L'individu considère les relations individuelles en termes de place dans le système. Il pense que la justice - mais encore une fois on peut en dire autant de la citoyenneté - consiste à accomplir les devoirs effectifs auxquels on a souscrit. Le contrat social et les devoirs qu'implique le fait de l'avoir accepté sont des moments décisifs. Il s'agit alors essentiellement d'apporter son soutien aux lois (sauf cas extrêmes). Or c'est très exactement l'attitude de nos « légalistes ». Ils prônent l'obéissance aux lois dans tous les domaines, par exemple ils sont significativement les plus défavorables à ce que, selon les termes du questionnaire, "les citoyens puissent entreprendre des actes de désobéissance civile pour s'opposer aux actions du gouvernement $\gg$.

La logique qui préside à la formation de notre troisième type, celui des « individualistes indépendants », est la moins facile à identifier. Toutefois, sa proximité avec certains aspects du stade 5 de la théorie de Kohlberg peut justement s'avérer éclairante. Rappelons qu'avec ce stade on entre dans la dimension post-conventionnelle où les décisions morales sont régies par des principes. Comme les «légalistes » du stade 4, les individus du stade 5 apportent leur soutien aux institutions et aux lois, y compris dans des cas de conflits entre ces normes et les règles concrètes du groupe social dont ils sont issus. Nous retrouvons cette parenté dans nos données au travers du fait que légalistes et individualistes se situent du même côté de l'axe 2 horizontal de notre ACP. Il n'est en conséquence pas question d'un individualisme « débridé » ou en quelque sorte anarchiste. Cependant, au stade 5, les individus prennent en considération le point de vue moral et le point de vue légal, reconnaissent leurs divergences et estiment qu'il est difficile de les concilier. La légitimité des normes perd à leurs yeux l'évidence qu'elle avait aux stades précédents, et cela ne va pas sans susciter l'exigence de leur justification. Pour que cette justification soit possible, et comme nous n'en sommes pas 
encore au principe d'universalité qui viendra ultérieurement, il faut bien alors au minimum qu'il soit légitime que l'individu puisse faire valoir son point de vue en toute indépendance. La dénomination d' ' individualistes indépendants » que nous avons finalement retenue tient à ce profil spécifique de personnes qui valorisent à la fois le fait de voter comme celui d'être attentif à l'action gouvernementale mais qui ont également, comme le montrent des croisements avec d'autres questions de l'enquête, une nette propension à parler politique avec leur entourage et à vouloir le convaincre du bien-fondé de leurs positions. Elles appartiennent souvent à des associations et pensent, dans beaucoup de pays, que le bon citoyen devrait participer à la vie associative. L'individualisme ici revendiqué ne doit donc pas s'entendre comme une indifférence à l'égard de la loi - comme le montre la proximité avec les « légalistes »-ou plus largement du politique, mais plutôt comme une indépendance quant à l'appréciation de tout ce qui touche au politique et à la légitimité des normes. Le citoyen idéal exprime ses opinions et exerce son regard critique.

Le choix que ces individus font de trouver important de «toujours voter aux élections » aurait certes pu recevoir une interprétation «conformiste »: on respecte cette norme civique comme on obéit aux lois. C'est d'ailleurs le cas au Japon où cet item se regroupe avec ceux de l'obéissance aux lois. Mais il s'en détache dans les autres pays pour s'associer avec l'attention qu'il convient de porter à ce que fait le gouvernement, ce qui signifie que le vote y est davantage vu comme une occasion à ne pas manquer d'exercer son regard critique. Il s'agit moins de simplement accomplir un devoir civique que d'encourager à toujours se saisir, ou le plus possible, de tous les moyens d'exprimer son opinion. Dans le même esprit, "porter attention aux actions du gouvernement » peut s'interpréter comme une possibilité, pour les agents, d'actualiser leur capacité à juger de la légitimité de l'action gouvernementale ou législatrice en rapport à un principe moral auquel ils adhèrent. Trouver cet item important ne nous semble pas strictement signifier que l'enquêté valorise simplement le fait de se tenir informé, d'écouter la radio ou les informations grand public. Il s'agit bien plutôt d'exprimer une attitude critique, d'une certaine manière comparable à celle sous-jacente au stade 5 de la conscience morale identifié par Kohlberg.

Comme le rappelle l'annexe, le stade suivant et ultime, le stade 6, est toujours régi par des principes. En ce sens il reste post-conventionnel, mais il s'agit cette fois de principes éthiques universels. Autrement dit, les décisions morales y sont le fruit de principes, qui font ou pourraient faire, l'unanimité. A ce stade, l'individu reconnaît la pluralité des conceptions du bien et ne cherche pas à la réduire au nom d'une conception du bien particulière. Il recherche, de manière libérale, l'accord de tous les concernés sur des principes de justice équitables «purement procéduraux », pour utiliser le vocabulaire de Rawls, et qui permettent en raison de ce caractère purement procédural à ces différentes conceptions du bien de pouvoir vivre ensemble ou de faire société. C'est ce qu'il faut entendre par «raisonnable» (Forsé et Parodi, 2004) et l'on voit pourquoi ce stade est décrit par Kohlberg comme celui de la morale formaliste (= procédurale). Pour la mettre en œuvre, les sujets agissent en s'orientant vers l'inter-compréhension et déploient une compréhension décentrée du monde.

Or c'est précisément ce qui, dans nos données, caractérise les «pluralistes libéraux » (quatrième et dernier groupe de notre typologie) puisque selon eux un trait fondamental du citoyen idéal est d'" essayer de comprendre les raisonnements des gens qui ont d'autres opinions » tout en étant par ailleurs capable de «choisir certains produits pour des raisons politiques, éthiques ou environnementales, même s'ils coûtent un peu plus cher». La proximité de ces deux manières d'envisager le citoyen idéal suffit à montrer que le pluralisme dont ces enquêtés font preuve ne peut s'entendre en un sens relativiste suggérant qu'ils s'intéresseraient à tout parce que tout se vaut. Bien au contraire, le type dont il s'agit ici est celui d'un « libéralisme raisonnable » qui n'est pas prêt à accepter n'importe quoi, comme le montre aussi la proximité de ce type avec l'item "porter attention aux actions du 
gouvernement ». Le «pluraliste raisonnable » n'entend pas accepter ce qui remet en cause le raisonnable au nom d'on ne sait quelle liberté infinie. Au lieu de cela, la morale se fonde pour lui fermement sur le principe de l'égale dignité d'autrui. Le souci d'autrui, en l'occurrence de ses jugements, n'est donc pas l'apanage des seuls « altruistes ». Il vaut pour ces pluralistes qui se situent pour cette raison du même côté de l'axe horizontal de l'ACP que ces altruistes. Toutefois, pour les pluralistes, le respect d'autrui se réalise en considérant l'autre comme une fin plutôt que comme un moyen et par conséquent dans l'effort pour s'intéresser à ses opinions et les comprendre. S'actualise ici un souci réflexif pour autrui qu'ignorait l'altruisme davantage fondé sur la prise en compte des affects du prochain.

Au total, sans prétendre vouloir apparier trop strictement chaque type de citoyen idéal avec un stade de la description kohlbergienne du développement de la conscience morale, la référence à cette théorie permet bien de conférer un surcroît d'intelligibilité aux réponses données par les enquêtés. En particulier, le couple conceptuel «conventionnel vs. postconventionnel » donne effectivement sens à la répartition typologique des enquêtés dans le plan factoriel. Ainsi, par exemple, ce que nous avons jusqu'ici nommé "rapport à autrui » pourrait sûrement tout aussi bien être désigné par le terme «solidarité ». Si cette solidarité est bien alors une composante essentielle de la définition du citoyen idéal selon nos contemporains, notre typologie montre cependant qu'elle revêt deux formes différentes. L'une, conventionnelle, fait appel à un altruisme et elle est en quelque sorte une question pragmatique. L'autre, post-conventionnelle, relève d'un pluralisme libéral selon lequel la solidarité des citoyens est bien plutôt à mettre au fondement du contrat social. C'est, rappelons-le, la position de Fichte (1796-1797, p. 224) et, partant, des solidaristes (Bourgeois 1896 ou Bouglé 1924) mais c'est aussi celle de Apel (1994, p. 42) qui ne cesse de répéter que du point de vue de l'éthique de la discussion les sujets sont co-responsables, autrement dit fondamentalement solidaires de leurs jugements ou décisions. C'est encore la raison pour laquelle Habermas (1989-1990), critiquant d'ailleurs Kohlberg sur ce point, tient à montrer que la justice au stade 6 relève de la solidarité et non de la bienveillance.

Ces résultats et leur interprétation ne sont pas forcément éloignés de ceux obtenus au travers d'autres recherches sur les valeurs et qui utilisent un lexique plus ad hoc. Notre typologie présente par exemple des traits similaires à certaines des valeurs de base recensées par S. H. Schwartz (2006) au travers des enquêtes qu'il a analysées. En particulier, les valeurs de la conformité, de la bienveillance, de l'universalisme, de l'autonomie ne sont pas sans lien, respectivement, avec les types conformiste, altruiste, pluraliste libéral et individualiste indépendant. Les valeurs de la conformité sont décrites par Schwartz comme issues de la nécessité pour les individus, d'inhiber les désirs qui, en eux, contrarieraient ou entraveraient le bon fonctionnement des interactions et du groupe. La bienveillance est conçue comme le souci du bien-être des autres. Elle est notamment associée au fait d'être secourable et loyal. L'universalisme qui s'exprime dans l'attitude de nos «pluralistes libéraux » tend, dans la liste de Schwartz, vers la compréhension, l'estime, la tolérance, la protection du bien-être de tous et de la nature. Enfin notre description des «individualistes indépendants » entretient quelque rapport avec ce que Schwartz définit par des objectifs d'indépendance de la pensée et de l'action, quand il cherche à caractériser (en commettant d'ailleurs une confusion) la valeur de ce qu'il nomme " autonomie ». Notre typologie ne reflète bien entendu pas l'ensemble des valeurs caractérisées par Schwartz puisque le spectre d'investigation qu'il s'est donné recouvre tout ce à quoi des personnes peuvent accorder de l'importance, alors que le nôtre est strictement délimité par une interrogation sur la seule citoyenneté, mais ces convergences partielles méritaient d'être signalées. 


\section{Sociodémographie des types de citoyen idéal}

Chacun des types de citoyen idéal que nous avons discerné peut bien sûr s'avérer être soutenu par des catégories sociodémographiques différentes. Il peut aussi davantage prévaloir dans certains pays que dans d'autres. De ce point de vue, il faut tout d'abord noter que, globalement, l'opinion politique et l'âge interviennent essentiellement dans le positionnement selon l'axe horizontal du graphique factoriel (cf. graphique 2). Les plus âgés et ceux qui se situent politiquement à droite se soucient surtout du rapport aux normes dans leur définition du bon citoyen, alors que les jeunes et les personnes de gauche optent pour le rapport à autrui. De leur côté, le diplôme, l'auto-positionnement dans la pyramide sociale, le sexe et la participation sociale jouent surtout un rôle eu égard à la position le long de l'axe vertical. Les hommes, les plus associatifs ou diplômés et se positionnant haut dans la hiérarchie sociale, adhèrent aux types post-conventionnels, tandis que les types conventionnels recueillent l'adhésion des femmes, des moins associatifs ou diplômés et de ceux qui se situent euxmêmes à des échelons peu élevés dans la hiérarchie socioéconomique. Toutefois, à de rares exceptions près, lorsque l'effet d'une variable sociodémographique sur le type de citoyen idéal est significatif, il va à peu près dans le même sens dans tous les pays.

Graphique 2 - Positions des variables sociodémographiques et des pays dans l'ACP des questions relatives au bon citoyen (axes 2 et 3 )

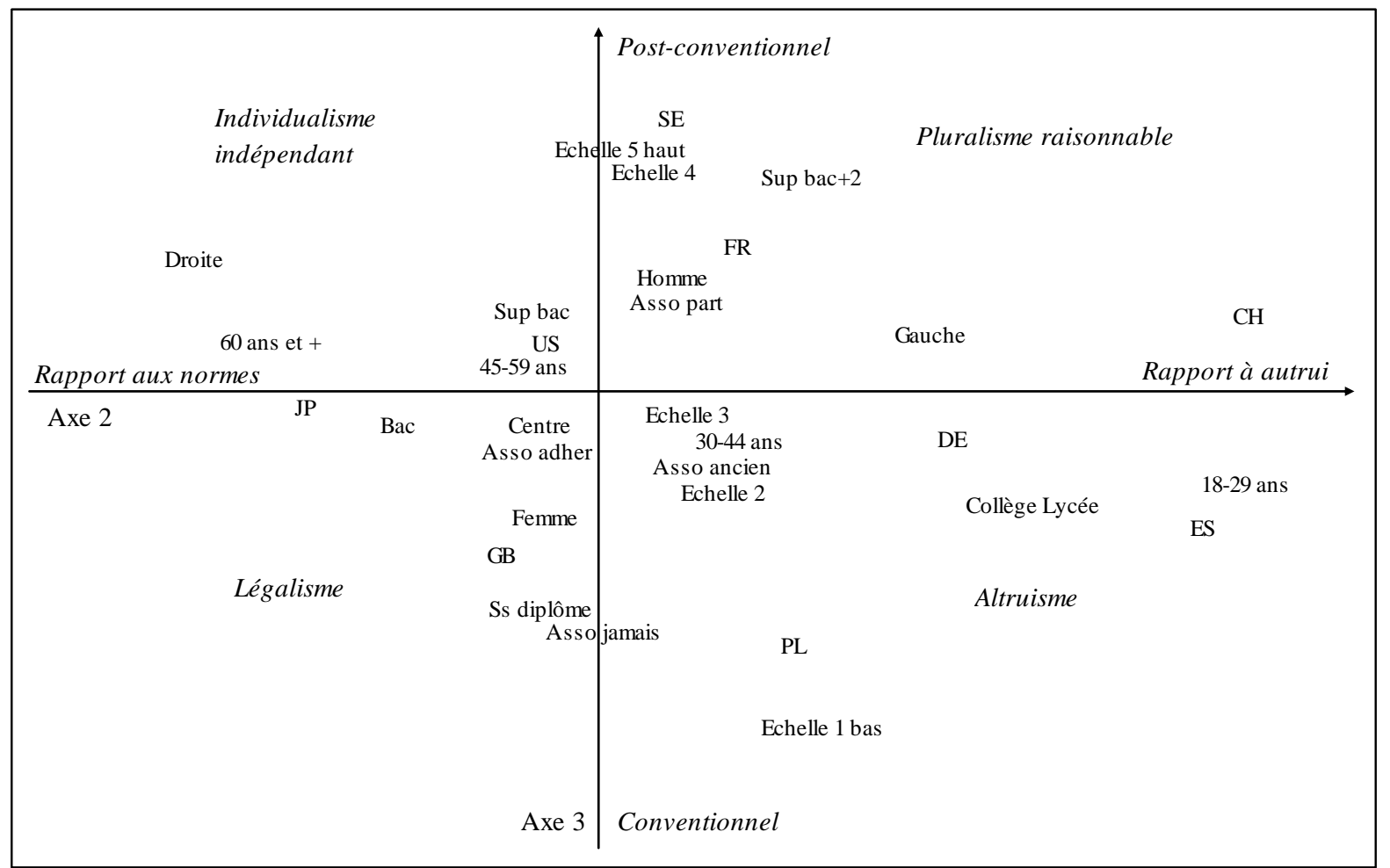

NB : Il s'agit de l'ACP représentée au graphique 1 à laquelle sont ajoutées, en variables supplémentaires, les catégories sociodémographiques et les pays. Les variables d'opinions sont les mêmes et restent seules actives dans la formation des axes. Toutefois, pour éviter la surcharge, nous ne les avons pas fait figurer ici. Les significations attribuées aux axes et aux quadrants sont en italique. L'abréviation "Echelle» fait référence à l'autoposition dans la hiérarchie socioéconomique. « Asso part » désigne ceux qui participent à au moins une association, «Asso adher », les simples adhérents, « Asso ancien », les anciens adhérents, et « Asso jamais », ceux qui n’ont jamais adhéré à une association.

Les particularités locales résultent davantage de ce que tel effet n'est pas significatif dans tel ou tel pays alors qu'il l'est d'autres. C'est ce qui explique l'essentiel des différences entre pays représentées au graphique 2 qui, d'ailleurs, ne se laissent associer, et c'est aussi un résultat, à aucun clivage selon un indicateur agrégé de type macroéconomique ou macrosocial (PIB, fécondité, religion, etc.). Même si, dès lors, ces différences ne doivent pas être sur- 
interprétées, on remarque que l'Espagne et la Pologne se reconnaissent davantage dans l'altruisme, tandis que la France, la Suède et la Suisse penchent plutôt pour le pluralisme. L'Allemagne hésite entre les deux. Le légalisme séduit davantage les Britanniques, alors que les Américains, bien qu'occupant la position la plus centrale, penchent en faveur de l'individualisme. Les Japonais sont à mi-chemin. Ce sont eux cependant qui font le plus intervenir le rapport aux normes pour juger du bon citoyen, alors qu'à l'opposé (sur l'axe horizontal) les Suisses sont ceux qui se soucient le plus du rapport à autrui. Selon l'autre dimension (axe vertical), les Polonais sont les plus conventionnels, tandis que les Suédois sont les plus post-conventionnels.

\section{Types de citoyen idéal et démocratie participative}

Ces différences étant notées, il reste à déterminer si ces manières d'appréhender le citoyen idéal ont des effets sur les attitudes à l'égard de la démocratie participative ou délibérative. En toute rigueur, ces deux termes ne sont d'ailleurs pas interchangeables. Ce que l'on nomme «démocratie participative » se distingue de la «démocratie délibérative », que l'on peut, en suivant A. Bächtiger et al. (2004), caractériser à partir de six critères procéduraux : les arguments qui y sont exprimés doivent être formulés en termes de «bien public»; les participants aux processus de discussion doivent exprimer leurs vues de façon authentique et vraie et doivent faire preuve de certaines qualités (comme écouter les arguments convoqués par les autres participants, traiter ces derniers avec respect); les arguments exprimés doivent procéder d'un échange d'informations ordonnées et de bonnes raisons; les participants doivent suivre la force du meilleur argument; chacun de ceux-ci participe à un niveau égal et sans contrainte à un processus politique ouvert. La «démocratie participative », en revanche, renvoie à une description beaucoup plus vaste de l'engagement dans la vie politique et publique, qui ne donne pas systématiquement lieu à une délibération ou à une prise de décision. Si la délibération a en général pour objectif de conduire à la décision (souvent par un vote), la simple participation ne l'exige bien sûr pas. Il faut cependant remarquer avec A. Bouvier (2007) que les auteurs qui traitent de ces questions ne distinguent pas toujours clairement entre participation et délibération et emploient même souvent un terme pour un autre.

Les questions posées dans l'enquête ISSP ne permettent de toute façon ni d'évaluer les traits qui viennent d'être évoqués ni d'envisager le détail des formes de participation délibérative et décisionnelle, en l'occurrence le débat consultatif ou la délibération aboutissant au vote. Nous nous serons donc contraints, pour cerner cette participation démocratique, de l'envisager en son sens large d'engagement dans la vie civique ou de participation à la prise de décision démocratique ${ }^{7}$, et non nécessairement au sens strict de participation à des débats publics et politiques ou à des processus de délibération.

Deux questions de l'enquête ISSP portent toutefois directement sur des opinions à l'égard de la démocratie participative. Bien qu'au premier abord elles puissent sembler proches, voire redondantes, elles sont en réalité assez différentes. La première interroge les enquêtés sur le fait de savoir s'ils jugent important (sur une échelle de 1 pour «pas important du tout» à 7 pour «très important») que «les hommes politiques tiennent compte des opinions des citoyens avant de prendre des décisions ». La seconde cherche à déterminer s'ils jugent important, toujours selon la même échelle, que «l'on donne davantage d'occasions aux gens de participer aux décisions publiques ». Dans le premier cas, la participation des citoyens peut être très indirecte (répondre à un sondage par exemple) et il revient aux hommes politiques de décider. Dans le second, on va davantage dans le sens d'une participation directe

\footnotetext{
${ }^{7}$ C'est également en ce sens large de participation à la vie politique publique que des auteurs comme N. Fraser (Fraser et Honneth, 2003, p. 232) exploitent la notion de « démocratie participative ».
} 
à la prise de décision elle-même, mais la formulation de la question n'exclut pas que les citoyens n'aient qu'une voix consultative. Au sens strict, il ne s'agit pas de démocratie délibérative, si l'on entend par là que les participants ont, à l'issue des débats, une voix délibérative (cas du référendum). Mais on peut parler de démocratie délibérative en un sens large, si l'on ne considère pas cette clause comme nécessaire. Cela va nous permettre de simplifier les expressions en parlant dorénavant, pour la première question, de « démocratie participative » (en un sens large) et, pour la seconde, de démocratie délibérative (également en un sens large).

Ces deux formes de démocratie font l'objet d'un consensus important. En moyenne, s'agissant de la démocratie participative, $71 \%$ des enquêtés se situent sur le plus haut degré de l'échelle d'appréciation et $14 \%$ sur le suivant. Ils sont respectivement $52 \%$ et $20 \%$ pour ce qui est de la démocratie délibérative. L'adhésion est donc un peu moins élevée (-13\%) dans ce second cas, mais elle reste tout de même de grande ampleur (sauf en Suisse où elle n'atteint plus que $48 \%$ ). Ce profil général vaut pour tous les pays. Il n'est dès lors pas surprenant que les réponses à ces questions soient fortement corrélées. En tenant compte de leur nature ordinale, le coefficient Gamma vaut en moyenne 0,77, ce qui représente une valeur élevée et significative. Néanmoins, pour restreint que soit le différentiel d'adhésion, il laisse supposer que ce ne sont pas exactement les mêmes catégories d'enquêtés qui privilégient un type ou un autre de démocratie. Il est donc légitime de se demander si les différents jugements sur le citoyen idéal ont un impact sur les opinions quant à ces formes de démocratie. L'adhésion à tel ou tel type de citoyenneté idéale conduit-elle à pencher plutôt en faveur de la participation ou de la délibération?

Certes, ces deux questions ne sont pas toujours significativement liées aux types de citoyens idéaux, notamment en France, en Allemagne et dans une moindre mesure aux EtatsUnis, et lorsqu'elles le sont, les liaisons ne sont pas strictement identiques partout. Il apparaît cependant que si les pluralistes ont tendance à rejeter la démocratie participative, ce sont parfois les individualistes qui la soutiennent (en particulier aux Etats-Unis). Mais surtout, la démocratie délibérative rencontre plus souvent l'adhésion des pluralistes (sauf en Suisse), voire des altruistes, tandis qu'elle est plutôt rejetée par les légalistes (sauf encore une fois en Suisse). Ceux qui, dans leur appréhension du citoyen idéal, mettent l'accent sur le rapport à autrui ont donc tendance à privilégier la démocratie délibérative ${ }^{8}$.

Bien entendu, les catégories sociodémographiques qui soutiennent le plus la démocratie participative ou délibérative correspondent à celles qui sont les plus impliquées dans les types de citoyens idéaux dont les rôles viennent d'être soulignés. Il n'est donc pas nécessaire de s'y attarder plus avant. On remarque toutefois qu'une participation sociale active conduit davantage à adhérer à la démocratie participative et à ne pas considérer que la démocratie délibérative soit importante.

Il est vrai qu'être actif dans au moins une association et plus particulièrement dans une association politique est d'abord le fait des individualistes, le plus souvent suivis par les pluralistes. Les légalistes ou les altruistes ne manifestent que peu d'enthousiasme pour ce type d'association et même pour la participation sociale en général, mais il faut rappeler que les associations à caractère humanitaire ne font pas partie de la liste présentée aux enquêtés.

D'autres formes d'engagement dans une citoyenneté active leur étaient proposées. D'abord sous l'angle de quatre types possibles de protestation se situant peu ou prou dans le cadre d'une action collective : 1 - signer une pétition, 2 - boycotter ou délibérément acheter

\footnotetext{
${ }^{8}$ Ils ne vont pas pour autant jusqu'à approuver beaucoup plus que les autres la procédure référendaire ( $«$ comme un bon moyen de décider lorsque des décisions politiques importantes sont enjeu », pour reprendre les termes du questionnaire). Ce résultat est toutefois assez normal car, dans le cas particulier du référendum, le niveau d'approbation est tel, $90 \%$ en moyenne avec un faible écart selon les pays, qu'il semble ne plus y avoir place pour une opinion différentielle, quel que soit d'ailleurs le critère de différenciation retenu.
} 
certains produits pour des raisons politiques, éthiques ou environnementales, 3 - avoir pris part à une manifestation, 4 - assister à une réunion ou à un rassemblement politique. Dans chaque cas, il leur était demandé de situer leurs réponses sur une échelle destinée à refléter quatre degrés d'investissement: 1 - l'avoir fait dans l'année écoulée, 2 - il y a plus longtemps, 3 - ne pas l'avoir fait mais être susceptible de le faire dans le futur et 4 - ne l'avoir jamais fait et ne pas envisager de le faire à l'avenir.

Or, quelle que soit la forme de protestation envisagée, elle est d'abord le fait des pluralistes, suivis des individualistes. Les légalistes rejettent ces types de contestation, parfois rejoints, mais à un degré moindre, par les altruistes.

Quatre autres manières de citoyenneté active étaient encore proposées aux interviewés (avec la même échelle de réponse). Elles sont cette fois moins directement liées à une protestation collective et permettent une action individuelle plus orientée vers l'expression de son opinion : 1 - contacter ou essayer de contacter un homme politique ou un haut fonctionnaire afin de lui exprimer ses opinions, 2 - donner de l'argent ou collecter des fonds pour une activité sociale ou politique, 3 - contacter les médias ou y apparaître pour exprimer ses opinons, 4 - participer sur Internet à un forum ou à un groupe de discussion politique.

Les pluralistes continuent d'être les plus engagés dans ces formes d'expression, mais les individualistes les talonnent de beaucoup plus près. Il s'agit là en effet d'exprimer son point de vue et c'est pour eux un devoir du citoyen idéal. Comme précédemment, les légalistes rejettent plutôt ces pratiques. Ils sont en cela suivis, quoique dans une moindre mesure, par les altruistes sauf, bien sûr lorsqu'il s'agit de collecter ou de donner des fonds pour une activité sociale ou politique où, cette fois s'ils ne l'ont pas fait, ils se disent enclins à pouvoir le faire à l'avenir.

Il faut souligner que s'il y a bien quelques variations d'un pays à l'autre, ces liaisons d'ensemble se retrouvent le plus souvent dans chaque cas. Nous sommes ici en présence d'une forte régularité dans les relations entre ces formes de citoyenneté active et les types de citoyen idéal. La dimension post-conventionnelle favorise nettement ces manières de participation citoyenne, alors que la dimension conventionnelle conduit à ne pas ou peu y souscrire. Les pluralistes sont les plus enclins à adhérer à ces pratiques mais, lorsqu'elles se font moins collectives et visent à promouvoir une expression plus personnelle, ils sont tout naturellement rejoints par les individualistes.

\section{Conclusion}

L'hypothèse du lien que nous cherchions à tester entre les manières d'envisager les devoirs du citoyen et les attitudes à l'égard de la démocratie participative est donc largement confirmée par les données. Dans tous les pays étudiés ici, les jugements normatifs à l'égard du citoyen idéal se répartissent, de manière stable, en quatre grands types selon que l'accent est mis sur le rapport aux normes ou aux autres et selon que la morale qui préside à ces jugements relève d'une forme conventionnelle ou post-conventionnelle. Mais toutes ces manières de dessiner le visage du bon citoyen ne conduisent pas à approuver identiquement les engagements dans la vie publique ou politique. Si une participation souple ou moins exigeante n'apparaît pas significativement liée à un type ou un autre de citoyenneté, en revanche une participation nettement plus active, voire confinant par certains aspects à une démocratie délibérative, semble exiger l'adhésion à une morale post-conventionnelle. Selon la première forme de cette morale, les individualistes indépendants se contentent toutefois de valoriser tout ce qui, dans la participation citoyenne, permet l'expression de soi ou de son regard critique, tandis que selon la seconde les pluralistes libéraux vont au-delà et s'avèrent finalement être les principaux soutiens d'une démocratie à caractère délibératif (même si, compte tenu du questionnaire, ce n'est pas ici au sens strict du terme). 
Au total, les conditions d'un consensus par recoupement, telles que posées par Rawls, sont atteintes dans les conditions libérales qu'il lui assigne. Une fois le voile d'ignorance levé, chacun peut trouver, dans sa conception du bon citoyen, des raisons de soutenir une éthique publique démocratique en tant qu'adhésion à un idéal de la raison publique. Rappelons en effet que chacune des dimensions de la citoyenneté (idéale) est en elle-même jugée importante, voire très importante, par les enquêtés. Ils se distinguent par la priorité qu'ils confèrent à l'une sur l'autre mais aucun d'entre eux ne nie le rôle central des devoirs de civilité qui incombent au citoyen. En revanche, l'usage public effectif de la raison, qui est aussi requis comme le soutient Habermas, sous la forme de la participation active aux diverses instances de la démocratie délibérative, n'est pas quant à lui identiquement pratiqué ou valorisé. Elle requiert l'adhésion à une morale post-conventionnelle et surtout, au sein de celle-ci, à un pluralisme libéral. 


\section{Annexe : Les stades de la conscience morale selon Kohlberg}

Les travaux de Kohlberg se situent dans le prolongement de ceux de Piaget (1932). Il s'agit comme chez ce dernier de rendre compte de la maturation du jugement moral. Piaget avait déjà montré qu'entre la petite enfance et l'adolescence les sujets qu'il avait observés passaient de l'hétéronomie à l'autonomie selon une séquence relativement invariante. Kohlberg revient sur cet axe principal, non pour le remettre en cause, mais pour mieux le spécifier. Sous un angle plus méthodologique, il s'appuie lui aussi sur des observations et des enquêtes empiriques. Mais il cherche à donner une portée autant phylogénétique qu'ontogénétique à sa théorie du développement moral selon différents stades et s'appuie aussi sur des données collectées auprès d'adultes.

Les deux premiers stades relèvent de ce qu'il nomme le «pré-conventionnel ». Au stade 1 de la punition et de l'obéissance, le droit est conçu comme une obéissance littérale aux régies et à l'autorité. L'enfant cherche à éviter d'être puni. Au stade 2, il est motivé par la poursuite de son propre intérêt et de ses propres besoins. Il conforme son jugement à des règles en vue de servir ses objectifs.

Le stade 3 marque l'entrée dans la dimension «conventionnelle». Il ne s'agit plus seulement de respecter des règles mais de répondre aux attentes de ses proches et des partenaires de l'interaction. Il s'agit d' « être bon », c'est-à-dire d'avoir de bonnes intentions et de se sentir concerné par les autres. La dimension du rapport aux autres oriente la façon dont les agents envisagent les normes.

Au stade 4, qui demeure conventionnel, l'individu parvient à une considération élargie de l'altérité en considérant sa communauté. Son jugement moral se formule alors dans un souci de soutien des institutions et de préservation de l'ordre social. Il œuvre au bien-être de la communauté en étant porté à préserver le fonctionnement des institutions.

Avec le stade 5, il entre dans la dimension que Kohlberg qualifie de «postconventionnelle». Cette fois, il tient compte de principes et ceux-ci peuvent ne pas être parfaitement adéquats avec ce qui se dégage des normes, lois ou institutions existantes. Aux stades post-conventionnels apparaît donc une réflexion critique sur la légitimité des normes. $\mathrm{Si}$ au stade 5 l'individu conserve une attitude de respect de la loi ou de la règle en vigueur, il s'engage également à reconnaître les droits fondamentaux tout en endossant à titre personnel les valeurs et opinions issues des traditions communautaires auxquels il adhère. Le stade 5 est dès lors le lieu de conflits possibles entre, d'un côté, les lois, les valeurs et les droits fondamentaux et, d'un autre, les normes sociales adoptées par chacun au nom de son appartenance communautaire. Si tel est le cas, les individus sont plutôt enclins à donner une priorité aux valeurs, aux droits fondamentaux et aux contrats légaux sur les règles et les lois concrètes du groupe. Il n'en reste pas moins qu'ils n'hésitent pas à exprimer leurs principes et de cette manière à souligner la légitimité de leur indépendance de jugement en tant qu'individus. Pour Kohlberg, cela se manifeste par le fait qu'ils adhèrent à un contrat social de type utilitariste - type qui se fonde justement sur l'indépendance et non l'autonomie de chacun, comme A. Renaut (1989) l'a par ailleurs bien montré.

Le stade 6 - second stade post-conventionnel en même temps que phase ultime du développement de la conscience morale - est celui des principes éthiques universels, de la morale formaliste, au niveau duquel le jugement moral prétend légitimement à l'universalité. Ici les principes éthiques prévalent sur les lois particulières. On constate également qu'à ce stade une place est faite au souci d'autrui aussi bien dans l'approche intellectuelle du monde par les individus que dans leur comportement. Mais la morale se fonde ici sur l'égal respect ou dignité d'autrui. Kohlberg note la parenté avec l'impératif catégorique (Kant, 1785). Le sujet observé est capable, grâce à une adoption réversible de rôles (concept largement 
emprunté à Mead, 1934), d'un décentrement tel qu'on pourrait tout aussi bien dire qu'il raisonne comme derrière un voile d'ignorance (Rawls, 1971). C'est ce qui conduit Habermas (1983) ou Apel (1986) à voir là un stade où les raisonnements sont proches de ceux d'un sujet qui ferait siens les principes de l'éthique de la discussion.

Il faut noter que le passage d'un stade à l'autre consiste, selon Kohlberg, dans une maturation morale progressive de l'individu : les stades sont hiérarchisés entre eux. Un même sujet expérimental ne peut régresser d'un stade à un stade inférieur pas plus qu'il ne peut, au cours de son développement, sauter un stade. Chaque structure cognitive doit être interprétée comme étant le résultat d'une réorganisation créatrice d'un équipement cognitif disponible qui, face à des problèmes qui se répètent, s'avère dépassé. Les structures cognitives d'un stade dépassent celles du stade inférieur, autrement dit, les remplacent tout en les préservant, dans une forme réorganisée et différenciée. Pour l'interprétation de notre typologie, et pour des raisons précisées dans le corps du texte, nous ne retenons notamment pas cette idée d'une évolution irréversible sous forme de stades. 


\section{Références}

Apel K.-O., 1994, Ethique de la discussion, Paris, Cerf.

Apel K.-O., 1986, Fondation de l'éthique de la communication [...], tr. fr. in 1998, Discussion et responsabilité, (tome II), Paris, Cerf, 85-133.

Audard C., 2007, Ethique publique et démocratie délibérative, in A. Benmakhlouf, Le lien social, Casablanca, Editions Le Fennec, 73-91.

Bächtiger A., Spörndli M., Steiner J. et Steenbergen M. R., 2004, Deliberative Politics in Action. Analysing Parliamentary Discourse, Cambridge (G-B), Cambridge University Press.

Bévort A., 2002, Pour une démocratie participative, Paris, Presses de Sciences Po.

Blondiaux L., Sintomer Y., 2002, L’impératif délibératif, Politix, vol. 15, n57, 17-35.

Bouglé C., 1924 ( $2^{\text {ème }}$ éd. entièrement refondue), Le solidarisme, Paris, Marcel Giard.

Bourgeois L., 1896, reprint 1998, Solidarité, Villeneuve d'Ascq, Presses Universitaires du Septentrion.

Bouvier A., 2007, La dynamique des relations de confiance et d'autorité au sein de la démocratie dite "participative" et "délibérative", Revue européenne des sciences sociales, $\mathrm{t} . \mathrm{XLV}, \mathrm{n}^{\circ} 136,181-230$.

Fichte J. G., 1796-1797, tr. fr. 1984, Fondement du droit naturel, Paris, PUF.

Forsé M., Parodi M., 2004, La priorité du juste, Eléments pour une sociologie des choix moraux, Paris, PUF ; tr. angl. 2005, The Priority of Justice, Elements for a Sociology of Moral Choices, Bern, Peter Lang.

Fraser N., Honneth A., 2003, Redistribution or Recognition? A Political-philosophical Exchange, Londres, Verso.

Gutmann A., Thompson D., 1997, Democracy and Disagreement, Cambridge, MA, Harvard University Press.

Habermas J., 1983, tr. fr. 1986, Morale et communication, Paris, Cerf.

Habermas J., 1989-1990, Justice and Solidarity: On the Discussion Concerning "Stage 6", The Philosophical Forum, vol. XXI, n¹-2, 32-52.

Habermas J., 1997, Droit et démocratie : entre faits et normes, Paris, Gallimard.

Habermas J., Rawls J., 1997, Débat sur la justice politique, Paris, Cerf.

Joss S. (éd.), 1999, Considering the concept of procedural justice for public policy and decision-making in science and technology, Science and Technology. Science and Public Policy, Special Issue on Public Participation, 26- 5, 290-373.

Kant E., 1785, tr. fr. 1994, Fondation de la métaphysique des mours, Paris, GF-Flammarion.

Kohlberg L., 1981, The Philosophy of moral development, San Francisco, Harper \& Row.

Mead G. H., 1934, tr. fr. 2006, L'esprit, le soi et la société, Paris, PUF.

Piaget J., 1932, Le jugement moral chez l'enfant, Paris, PUF.

Pufendorf S. von, 1673, reprint tr. fr. 2002, Les devoirs de l'homme et du citoyen tels qu'ils lui sont prescrits par la loi naturelle, Caen, Presses universitaires de Caen.

Rawls J., 1971, tr. fr. 1987, Théorie de la justice, Paris, Seuil.

Rawls J., 1993, tr. fr. 2001, Libéralisme politique, Paris, PUF.

Renaut A., 1989, L'ère de l'individu, Contribution à une histoire de la subjectivité, Paris, Gallimard.

Schnapper D., 2000, Qu'est-ce que la citoyenneté ?, Paris, Gallimard, Coll. Folio.

Schwartz S. H., 2006, Les valeurs de base de la personne : théorie, mesures et applications, Revue française de sociologie, 47-4, 929-968.

Sen A., 2006, La démocratie des autres, Paris, Rivages poche. 
Urfalino Ph., 2007, «La décision par consensus apparent », Revue européenne des sciences sociales, t. XLV, $\mathrm{n}^{\circ} 136,40-70$.

\begin{abstract}
The analysis of the 2004 ISSP survey, limited to nine of the countries participating to this programme of representative opinion polls, shows that the way people consider the duties of a citizen are everywhere divided into four types, depending on whether the emphasis is put on norms or on others, and whether the morality governing normative judgements about the "good" citizen, is conventional or post-conventional. These different axiological directions are linked with attitudes toward participative democracy. Certainly, a weak participation does not assume the support of one type, rather than another, of ideal citizenship, and the duty of civility, which can be associated with democratic public ethics, is approved quite widely. But a much more active participation, closed in some regards to a deliberative democracy, seems to require post-conventional morality, especially when it is founded on liberal or reasonable pluralism.
\end{abstract}

\title{
Résumé
}

L'analyse de l'enquête de l'ISSP de 2004 dans neuf des pays participant à ce programme de sondages représentatifs montre que les manières d'envisager les devoirs du citoyen se répartissent partout en quatre grands types, selon que l'accent est mis sur le rapport aux normes ou aux autres et selon que la morale qui préside aux jugements normatifs sur le «bon» citoyen peut être dite conventionnelle ou post-conventionnelle. Ces différentes orientations axiologiques ne sont pas sans lien avec les attitudes vis-à-vis de la démocratie participative. Certes, une participation peu exigeante ne suppose guère de soutenir une forme ou une autre de citoyenneté idéale et le devoir de civilité, que l'on peut associer à une éthique publique démocratique, fait l'objet d'une approbation relativement consensuelle. En retour, une participation nettement plus active, voire confinant par certains aspects à une démocratie délibérative, semble exiger l'adhésion à une morale post-conventionnelle, notamment lorsqu'elle se fonde sur un pluralisme libéral ou raisonnable.

\section{Zusammenfassung}

Wie die Erhebung zeigt, die von der ISSP 2004 in neun der an diesem Programm repräsentativer Umfragen beteiligten Länder durchgeführt worden ist, lassen sich hinsichtlich der Art und Weise, wie die Bürger ihre Pflichten verstehen, überall vier Typen unterscheiden: der Akzent kann auf die Beziehung zu Normen oder auf die Beziehung zu den Anderen gelegt werden; die Moral, die den normativen Urteilen über den guten Bürger zugrunde liegt, kann konventionell oder post-konventionell sein. Diese unterschiedlichen wertmässigen Einstellungen haben einen Einfluss auf die Haltungen zur partizipativen Demokratie. Eine anspruchslose Form der Beteiligung erfordert kaum das Bekenntnis $\mathrm{zu}$ einer idealen Staatsbürgerschaft, und die Pflicht zum korrekten Umgang, die mit einer öffentlichen Ethik der Demokratie einhergeht, ist weitgehend unbestritten. Eine aktivere Beteiligung hingegen, die auf eine deliberative Demokratie zielt, erfordert die Zustimmung zu einer postkonventionellen Moral, insbesondere dann, wenn diese auf einem liberalen, vernünftigen Pluralismus beruht. 